OPEN ACCESS

Edited by:

Theodoros Damartzis, École Polytechnique Fédérale de Lausanne, Switzerland

Reviewed by:

Limei Shen,

Huazhong University of Science and Technology, China

Dimitrios Ipsakis,

Technical University of Crete, Greece

${ }^{*}$ Correspondence:

U. Tuzun

prof.ugur.tuzun@cantab.net

Specialty section:

This article was submitted to

Process and Energy Systems

Engineering,

a section of the journal

Frontiers in Energy Research

Received: 04 May 2020

Accepted: 09 July 2020

Published: 31 July 2020

Citation:

Tuzun U (2020) Artificial Intelligence Assisted Dynamic Control of Environmental Emissions From Hybrid Energy Process Plants (HEPP).

Front. Energy Res. 8:179.

doi: 10.3389/fenrg.2020.00179

\section{Artificial Intelligence Assisted Dynamic Control of Environmental Emissions From Hybrid Energy Process Plants (HEPP)}

\author{
U. Tuzun ${ }^{1,2,3 *}$
}

${ }^{1}$ Churchill College, University of Cambridge, Cambridge, United Kingdom, ${ }^{2}$ International Process Engineering Consultant, Weybridge, United Kingdom, ${ }^{3}$ U.K. Delegate, European Federation of Chemical Engineering (EFCE) Energy Section, EFCE Working Party on Mechanics of Particulate Solids, Paris, France

A model digital data processing platform is proposed based on "deep-learning" methodology that can be used to identify the emissions patterns from process plants with hybrid energy recovery and energy generation facilities. The non-invasive dynamic monitoring and correlation of particulate, VOCs and other greenhouse gas emissions from semi-batch and continuous process plants is demonstrated with use of neural encoding and pattern recognition using a multi-layer perceptron and multi-stack encoder configuration. A multi-layer environmental perceptron (MLEP) is developed based on the above analyses that aims to detect patterns of emission types, rates and concentrations as a function of variation of plant operational conditions and process variables. Four different task algorithms are constructed and are currently trained for use in (i) In-Plant Product Quality Control Domain and (ii) In-Plant Process Efficiency Target Control Domain. As a further consequence, environmental impact assessment is considered within the hazards and process safety frameworks that conventionally issue sanctions and penalize non-compliance with imposition of environmental levy scales rather than offering process improvement incentives. The latter is demonstrated to be possible by facilitating dynamic corrective action and hazard prevention using MLEP platforms should emission ceilings be frequently and/or periodically exceeded in 24/7 continuous plant operations. Potential applications of the MLEP (MLEP) are illustrated in the context of dynamic emissions control and abatement in hybrid energy process plants (HEPP) and combined power plants using process-integrated $\mathrm{CO}_{2}$ capture and storage schemes.

Keywords: digital platforms, network analysis, environmental sensors, emissions control, HEPP

\section{INTRODUCTION}

Industrial process emissions of greenhouse gases, VOCs and particulates are shown to represent some $20-30 \%$ of all environmental emissions in industrialized economies (see for example, Erickson and Brase, 2019). Furthermore, the International Energy Agency [IEA] (2018) estimates that urban areas currently account for over $67 \%$ of energy-related global emissions, which are expected to rise to $74 \%$ by 2030 ; see IEA Report (2018). It is also expected that $89 \%$ of the 
increase in $\mathrm{CO} 2$ emissions from energy use will be from developing economies by 2030. Hence, reducing greenhouse gas emissions and improving air quality are two interrelated challenges for world economies in combatting climate change and degradation of natural habitats; refer to Erickson and Brase (2019). 7th Environment Action Programme (7th EAP) mandated by EU aims to develop by 2018 "strategy for nontoxic environment that is conducive to innovation and the development of sustainable substitutes including non-chemical solutions"; refer also to European Environment Agency [EEA] (2016) National Emissions Ceilings Directive (2016/2284/EU) and the Clean Air Programme.

Figure 1 shows a schematic summarizing the three main categories of operations where adverse environmental impacts are to be minimized: (1) Abatement and/or capture of atmospheric emissions of fine particulates, volatile organic compounds, and other greenhouse gases during recovery of heat losses, and re-use and re-generation of power;. (2) further treatment of byproducts to yield value-added products and recycle and re-use of process waste in energy generation and/or raw material provision to other industry sectors; and (3) use of recyclable and/or bio-degradable packaging materials of commercial products.

All three categories of these activities combined now constitute essential parts of industrial plant design and operation to achieve best possible compliance with environmental regulations (see for example European Environment Agency [EEA], 2016). Best Available Techniques (BATs) advocated for Large Combustion Plants (L) with total rated thermal input equal to or greater than $50 \mathrm{MW}$ of electrical power and heat production. Clearly, once a plant is operational, it also becomes imperative to monitor environmental emissions regularly to minimize atmospheric pollution as well as monitoring possible accumulation over time of pollutants ejected to environmental land and water with a view to re-process waste streams containing pollutants of high toxicity detrimental to natural habitat and human health.

Many of the classical chemical process plant control algorithms are established to provide feed-back loops and differential and integral control of set-points of process variables evaluated from the process control parameters such as recycle rates, flow rates, concentrations and phase distributions of materials; followed by "pinch" techniques used to achieve final product purity whilst providing maximum heat recovery (see for example Smith, 2005) for a comprehensive account of industrial energy systems integration. However, these steady-state process control algorithms often lack transient response analyses required to monitor and control environmental emissions largely due to the existence of "hidden layers" of interaction of process variables affecting the values of the "visible" measurements recorded by digital environmental sensors.

A rather more sophisticated type of operator control is therefore needed based on "deep-learning" methodology which makes use of supervised and unsupervised neural network algorithms aimed at establishing multivariate pattern recognition with varying numbers of hidden layers of process variables, operational conditions and material properties (see Tuzun, 2017, 2018). Application of emissions bubble concept for emissions monitoring and control is illustrated more recently by Tuzun (2020b) using a case study from a multifeed option fuel production plant which also incorporates the integration of CAPEX and OPEX minimization whilst meeting regulatory emission targets set for the emissions bubble.

\section{MONITORING ATMOSPHERIC EMISSIONS AND PATTERN RECOGNITION}

Little process data is reported in the open literature on VOCs, particulate and other greenhouse gas emissions from individual process and power generation plants due to the regulatory implications discussed in the introduction. However, an analogy is established in terms of the process time between data reported on emissions from animal feedstock and agricultural waste storage silos of methane and the higher weight alcohols such as ethanol, and acetic acid and acetaldehydes and esters (see WendaPiesik et al., 2010; Hafner et al., 2013) for reported data sets. These data sets show high atmospheric release rates (typically, $0.5-5 \mathrm{~g} \mathrm{~m}^{-2} \mathrm{hr}^{-1}$ with an air velocity of $0.5 \mathrm{~m} \mathrm{~s}^{-1}$ ) of more volatile compounds which feature significantly in the relatively short exposure times (typically 10-30 min) of the field sensors. In contrast, much higher accumulated emission results are revealed for heavier organics over time periods of the order of several hours. Furthermore, the VOCs emissions produced by bacteria and yeasts during and post-anaerobic processes are strongly affected by high temperatures and humidity (see Wenda-Piesik et al., 2010).

Bent (2015) and Yue et al. (2017) provide an in-depth analysis of the sources of agricultural greenhouse gas emissions from farm production to food consumption. Bent (2015) provides links to literature on chemical routes to eliminate methane and $\mathrm{NO}_{x}$ by nitrification/de-nitrification ( $\mathrm{NO}$ to $\mathrm{NO}_{2} / \mathrm{NO}_{3}$ and ammonification $\left(\mathrm{NH}_{3}\right)$. Yue et al. (2017) present extensive statistics indicating that Carbon footprint from food supply side to be almost threefold of those on the food consumption side. Greatest source of methane emissions are noted to be from farming of crops and fertilizer applications as well as from the use of crops as feed to livestock. These confirm the previous observations of Bent (2015).

Greenhouse gas emissions such as $\mathrm{CO}_{2}$ and NOx and Methane feature more heavily in power generating plants and those process plants which run combined cycle gas turbines and waste heat recovery (see for example Zare and Mahmoudi, 2015; Zhang et al., 2019). Furthermore, large combustion plants and waste incinerators can generate as much as $46 \% \mathrm{CO}_{2}$ and $18 \%$ NOx by volume in process flue gases; refer to European Environment Agency [EEA] (2016) and IEA-GHG (2019). With greenhouse gas emissions, the atmospheric release rate is fast whilst the accumulated emission readings are influenced by the advance and expansion of plumes as a function of the stack heights of the burner units (see Markiewicz, 2006) for details of mathematical modeling. 


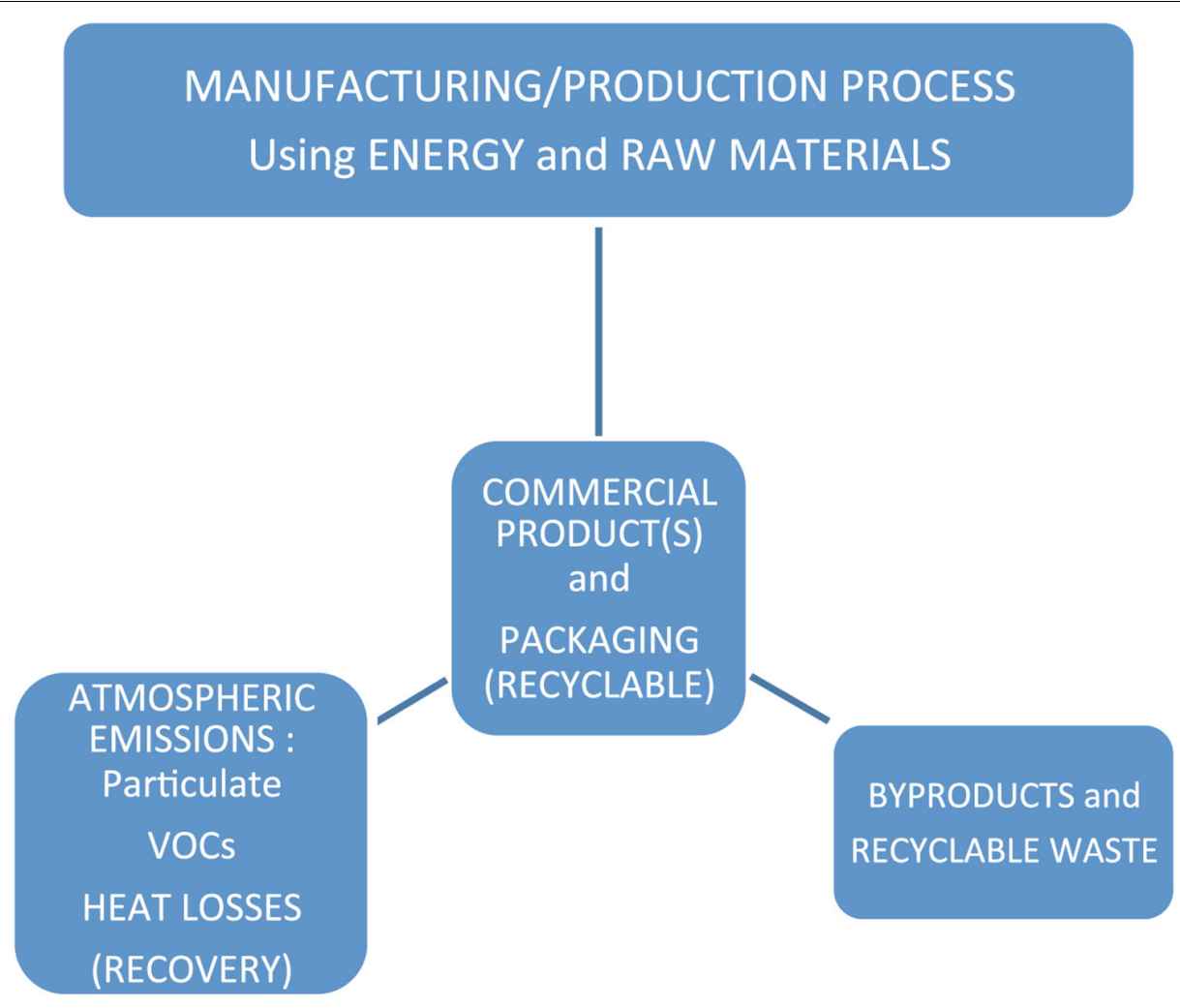

FIGURE 1 | Green process/production/manufacturing: recovery, and reuse of materials and energy and minimizing atmospheric emissions.

Particulate emissions with (PM-2.5) and (PM-10) micron limits are measured through online sampling around the particulate matter storage, handling and processing units (see for example, Kulp and Ponte, 2000). These emissions are shown to periodically peak and gradually disperse over time as a function of the operations performed by the batch, semi-batch and continuous operational units of the process plants.

Hence, in reliable monitoring of all three categories of emissions; VOCs, greenhouse gases and particulate matter, digital data gathering by multiple and integrated sensor systems become necessary; those that can self-correlate emission release rates, concentration profiles with simultaneous measurements of process temperature and relative humidity at the very least; refer to Figure 2 below for a schematic of environmental sensor platform used by Sensirion AG Switzerland. Such an integrated sensor and parallel emissions field data gathering platform could be used in the construction of the logistical (i) selection, (ii) identification, and (iii) data recording functions for a given HEPP application; refer to Figure 2.

Such an integrated environmental sensing platform is the essential pre-requisite for (i) demonstrating compliance with environmental health and safety regulations and emissions ceilings discussed above as well as (ii) facilitating the use of neural network analyses for time-series pattern recognition of atmospheric emissions from spatially distributed emission sources within and immediately adjacent to the combined process and energy cycle plants.

\section{DIGITAL PLATFORM CONSTRUCTION FOR MULTIVARIATE PROCESS AND ENVIRONMENTAL DATA SETS}

Environmental field sensors (refer to Figure 2) can be placed at different locations within potentially high-emitting plant sections or throughout the entire plant layout at specific spatial intervals. These sensors can be tuned to record data at set frequencies over varying time intervals and/or only when at levels above the non-compliance thresholds set by the operator. The dynamic process values measured of these process environmental variables are affected by each other. Accordingly, the fluctuations measured of one set of environmental sensing variable will/may affect the dynamic variations in the other environmental data sets. The interdependency of the dynamics of these environmental variables will reveal patterns in time-series and in spatially distributed data sets collected simultaneously from sensors placed at different locations within a process plant or indeed at different locations within a single large process unit/section.

\section{Trellis Diagram Construction}

In parallel to the environmental emissions data gathering, the process operator system will have (i) set point and acceptable limit specifications of a range of product stream quality parameters and (ii) process efficiency determinants of the process 


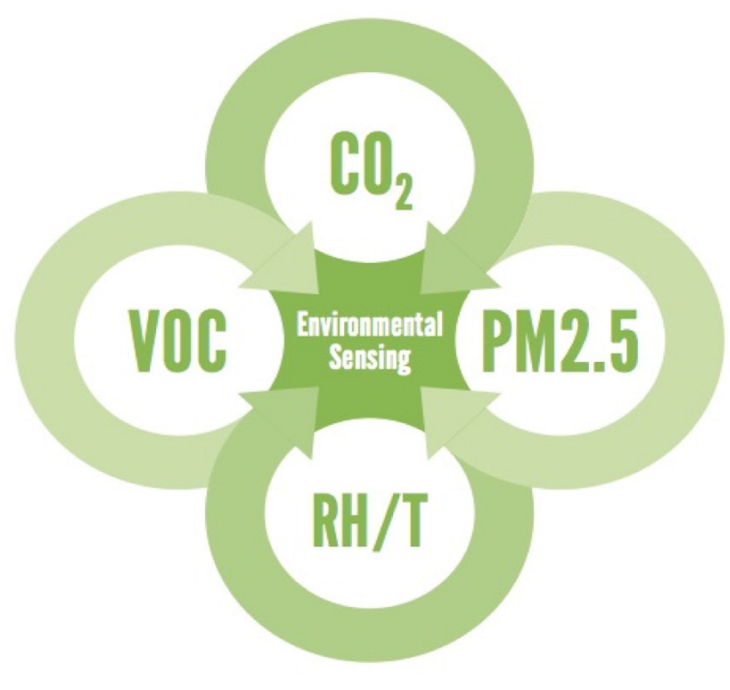

\section{SELECTION: Sensor Type \\ Location \& Population via \\ Emission Bubble(s) Identification within HEPP sub-sections}

\author{
2. IDENTIFICATION: Hybrid \\ Energy Input \& Output Flows \\ through successive unit \\ operations and intermediate \\ material storage and handling \\ stages
}

\section{DATA RECORDING: Spatial and Temporal Sampling Frequency in sync with process operating parameters and online quality control}

FIGURE 2 | Environmental sensing platform required for multiple detection and measurement.

unit operations. To connect the environmental sensor data to the process control data above, a Trellis Diagram of the pathways of possible connectivity between the "hidden" layers/units and the observed operator symbols can be generated based on the sequential and parallel stages of the process flowsheet (see Tuzun, 2018) for the demonstration of studies involving two and three hidden layer applications in cereal processing plants (Kulp and Ponte, 2000) and pharmaceutical process plants (see also Fujiwara et al., 2005). These will also be published in detail.

\section{Operator Tuning of "Preferred" States}

The use of input and output data convolutions produce large time-series data sets that can be correlated either by defining contrastive divergence or convergence to and from preferred states; e.g., future emissions targets. Alternatively, or even concurrently, it is also possible to make use of different permutations of connectivity of the hidden process layers to arrive at convergent and divergent patterns of causality of the spatial data sets connecting different process units and plant sections. This method of operative tuning would then also allow for the recognition of "peak events" and anomalies in time-series data. Consequently, connected process variable re-adjustment is made possible by direct operator intervention using environmental sensor data rather than using environmental sensor data purely as process safety alarms.

Figure 3 shows a conceptual schematic of an operator driven platform for environmental emissions minimization whilst maintaining appropriate operator thresholds of variability of process unit operations to achieve optimal process efficiency and product quality.

\section{Multiple Input/Multiple Output (MIMO) Multiplex Digital Platform Construction}

Environmental compliance of hybrid process and energy plants (HEPP) is best served by a multiplex platform for regular and simultaneous data transfer to enable the analysis of environmental impact on a continuous and operator interactive basis. Such dynamic statistical data processing is also potentially relevant to a significant number of other on-site operational efficiency assessments including (i) energy use and recovery; (ii) supply chain efficiency of raw materials and energy inputs; (iii) byproducts recovery; (iv) regeneration and reuse of process water. Hence, as well as providing the means for an efficient dynamic environmental impact control, such digital platforms can also be used effectively to decide and execute dynamic process improvements through corrective and preventive actions to be tried and tested by the interactive operator control. This could in turn lead to significant potential energy and material resource savings and waste minimization paving the way for green processing and clean technology applications commensurate with circular economy targets (see for example, Mendoza et al., 2017; Tisserant et al., 2017).

A tailor-made development and utilization of a Multiple Input/Multiple Output (MIMO) system and method are necessary that could facilitate environmental sensor data acquisition via a Multiplex Digital Platform. MIMO system and method are patented for use in wireless LAN and broadband data reception and transmission (see for example Jia and Julian, 2014; Sun et al., 2015).

MIMO system includes steps comprising (i) multiple sensor input streams, (ii) control value checks and noise pruning/elimination and data matrix inversion followed by 


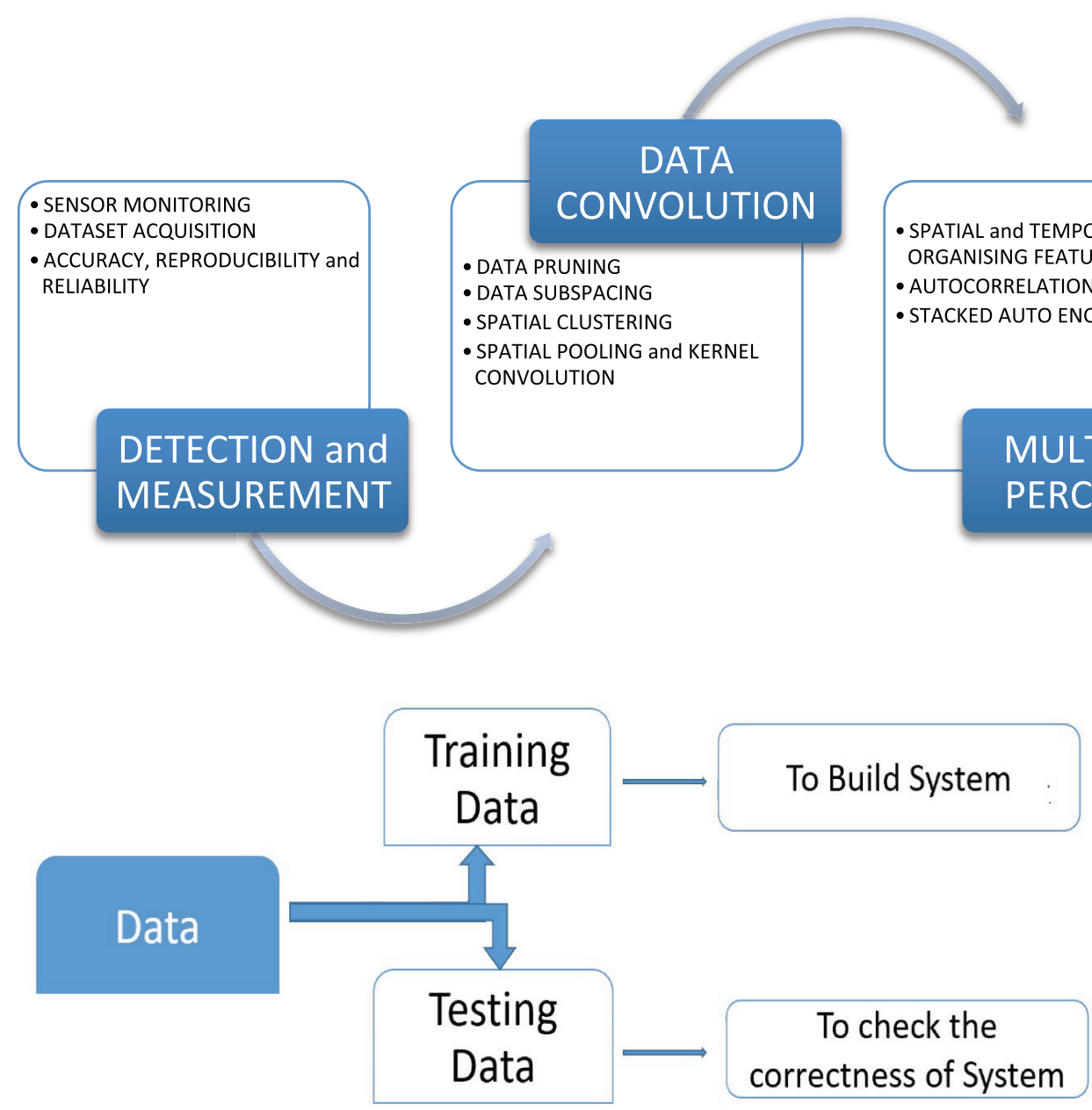

FIGURE 3 | (A) Construction of a digital platform for environmental data analysis: (i) multiple parallel sensor data gathering, (ii) spatial and Temporal Trend Evaluation through input and output data convolutions, and (iii) pattern recognition by unsupervised and supervised deep-learning. (B) Application and installation of the digital platform for environmental data analysis (i) Training: $4 \times$ task algorithm development in $2 \times$ test domains (ii) Testing: (i) emissions bubble(s), (ii) hybrid energy input and output energy flows. A digital platform for environmental data analysis: build-train-test methodology.

(iii) data stream splitting/data selection adding associated service descriptors (process plant topographic labeling); (iv) multiplex ENCODING of split and selected data sets for error correction in reconstructed data sets; and (v) digitization of the data sets for inter-leaver and modulator functions enabling the use of the VITERBI algorithm (see for example Lou, 1995; Wu, 2006). The use of the said algorithm allows the recognition of patterns by identifying and selecting the most likely sequence of "hidden states/layers" of process operational data which result in a given pattern of observed states/events; e.g., recorded time-series of air-borne emissions data sets at different plant sections.

The operations involving the use of the Viterbi algorithm are also referred to as DECODING the connectivity of the "hidden" operational layers of the process plant. Mathematical construction procedures of a Convolutional Neural Network (CNN) to enable output data decoding is described briefly below (see Haykin, 2008) for more details.

\section{NEURAL NETWORK ANALYSES OF MULTIVARIATE PROCESS DATA}

There are two types of mathematical approach to neural network applications known as unsupervised and supervised "Deep-Learning" techniques; they can be used separately, independently or simultaneously: (i) Unsupervised DeepLearning using iterative construction of self-organizing maps (SOM) of environmental sensor data clusters by producing affine transformations of spatial and temporal neighborhood sub-spaces of data of high similarity generated with customized distance metrics (see for example Langkvist et al., 2014; Antoniades and Took, 2016); (ii) Supervised Deep-Learning achieved through calculation of contrastive convergence and divergence of data from targeted outputs using connectivity of hidden layers (units/neurons) of a multi-layer perceptron (MLP); refer to section "Digital Platform Construction for 
Multivariate Process and Environmental Data Sets" and (see Haykin, 2008) for details.

In unsupervised deep learning, the mathematical model constructs input vectors that correspond to the "preferred state" of all sensors and controls. The convergence and divergence to and from the time-series patterns displayed with the spatial SOM grid of the "preferred state" can then be monitored by operator tuning of the process control parameters; refer to Figure 3. Iterative visual superimposition of real-life data sets on the SOM grid representing the preferred state of all sensors and process controls allows process control tuning in a manner, rather like using a virtual kaleidoscope.

\section{Previous Developments With CNN Applications}

It is also important to establish the extent of previous developments of the CNN algorithm construction and application in other diverse environmental process related fields of study. Here, by way of related examples, we will contrast the Multi-Layer Environmental Perceptron (MLEP) proposed here for monitoring and control of industrial plant emissions; with the works in computer-assisted electrical tomography for imaging building structures to monitor impacts of extreme weathering (see Rymarczyk et al., 2018); mapping scalp detectors activity to intracranial EEG for monitoring causality of epilepsy and aiding with clinical diagnosis (see Antoniades et al., 2018) and speech/speaker recognition in long temporal acoustic signal convolution contexts to model phonetic information; refer to Snyder et al. (2015). All these studies make use of the Convoluted Neural Network analysis based on the construction and training of algorithms on hidden-layers of connectivity as well as unsupervised pattern recognition techniques based on data pooling and sub-space mapping techniques; refer to Haykin (2008).

The above examples are cited because of their use of algorithm constructions with increasing order of sophistication. Long-time averaged spatial image construction with electrical tomography (Rymarczyk et al., 2018) relies on 2-D planar data filtered re-constructions from simultaneous voltage and capacitance readings from multiple sensors whilst the clinical data analysis with intracranial EEG analyses time series of data in individual channels and thus uses only 1-D filtered data sets; refer to Antoniades et al. (2018). In acoustic recognition algorithms, speaker recognition is established using i-vector mapping and Gaussian Mixture Models (GMM) (2-D) voice recognition with additional CNN algorithm addition a posteriori for alignment of phonetic content in tied 3-D states or senones used to define phonetic sub-space similarity against a trained reference state for advanced speaker identification with content; refer to Snyder et al. (2015).

It is clear from the examples provided above that the MLEP proposed for industrial emissions monitoring and abatement could use both spatial mapping and time-series re-construction analyses of environmental sensor data sets based on unsupervised mapping and supervised convolution techniques; refer to Figure 3. The MLEP is constructed to connect dynamically the three successive stages of dynamic process data analysis and process abatement: (i) detection and measurement, (ii) data convolution, and (iii) deep-learning for pattern recognition; refer to Figure 3A.

Finally, the supervised deep learning by CNN construction requires a priori training to be achieved with repeated modulation at different frequencies (i.e., different input data sampling intervals) of the hidden-layer algorithms using randomly generated field sensor data sets. This helps to establish the reference states; expressed as channel-labeled waveforms in a multi-channel data analyzer; for comparison with reallife data sets to enable operator fine-tuning of the process parameters affecting the environmental sensor readings. There is ample textbook literature and dedicated on-line sites for independent learning such as (adeveloperdiary.com) which also provide illustrations of sample software and case study demonstration packages.

\section{Novel Kaleidoscope Model of Process Environmental Compliance Control}

A novel KALEIDOSCOPE MODEL of a MLEP is currently being developed to facilitate pattern recognition with supervised deep learning. This is achieved by auto-correlation of data using alternate sequences of "hidden layers" and increasing the hierarchy of hidden layers by placing additional specs of variables in each layer. The early results indicate that it is possible to build up increased layers of multivariate and multi-stack encoder operation (refer to section "Operator Tuning of "Preferred" States" above). This will allow for greater degrees of freedom of operator control in fine-tuning of data acquisition by the digital environmental field sensors by continuous monitoring of the process dynamics; refer to Figure 2.

Further progress with successful implementation of the MLEP is likely to reduce the need for routine large-scale uncorrelated raw data storage and handling currently necessitated by the conventional "in situ" process measurements. This is expected to allow the plant operators to focus predominantly on the product quality and process efficiency targets.

Furthermore, the operational records produced by the MLEP enabling green process applications can in turn be used to design novel integrated environmental sensors that can collect and auto-correlate data directly, targeting environmental compliance performance of a hybrid energy process plant.

Figure 4 shows a conceptual schematic of the MLEP digital data processing platform comprising the two simultaneous machine learning cycles of input and output data convolutions and construction of SOMs, spatial clustering and time-series pattern recognition in reconstructed data. The operator interface will then be used to (i) reset noise filter parameters, (ii) refresh digital sensor output frequency thresholds, and (iii) re-adjust process parameter values to comply with the environmental emission ceilings on a dynamic basis. Figure 4 also illustrates the dynamic operator interface functions synced with the MLEP to recover and maintain the "preferred state" control of environmental emissions. It is expected that specific process applications will/may require multiple sets of hardware for 
operational control of different plant sections established to be potential high-emitters. This is believed to be in accordance with the "emissions bubble" concept approved by the European Environment Agency (EEA) Directives (2016-2284). This considers the weighted average of emissions of all emitters within the "bubble" for approval of regulatory compliance.

\section{Real-Life Training and Plant Trials With MLEP}

Generic training of the MLEP with different deep-learning algorithms such as Recurrent Neural Network (RNN) and TimeDelay Neural Network (TDNN) is designed and tested by producing simultaneous input and output data convolutions (see for example Haykin, 2008). It is possible to design information pathways connecting multiple "hidden layers" with operator observed data sets by selecting functional connectivity between the time series of key material sample properties and process variables for each unit operation in the process plant starting with the Trellis diagram constructions described in section "Trellis Diagram Construction." This is procedure is known as defining the learning rules which allows the neural network to maintain a sort of "state" facilitating such tasks as a sequence prediction. Training of the constructed pathways of connectivity with specific deep-learning algorithms is affected using plant operator and environmental emissions data sets.

The operational data training (as seen in Figure 3B) using the digital platform is affected by the development of 4 task algorithms: (i) estimation of variability of material flow rates, (ii) estimation of variability of emissions of different kinds, (iii) estimation of operational disruption rates and (iv) re-current anomaly detection and Environmental Neural Switch when spatial variability takes precedence over temporal variability and vice versa. Here, issues such as noise elimination, inputoutput signal time drift, and cumulative time-series pattern recognition indexed to sample reference states are computed to build up a reference library of the "known states" of the process plant operation.

In real-life plant operation, the contrastive convergence and divergence to and from the "known states" is recognized when the measured parameters and sequence-predicted values from the NN algorithms are kept within certain pre-set safe operating limits. When environmental emissions values rise significantly above the "preferred state," then this would allow HAZOP procedures to kick in at different levels of pre-set perceived risk levels. To date, a real-life demonstration of the automated linking of HAZOP and MLEP-based harmful emissions data predictions based on environmental sensor inputs is yet to be pioneered. The additional challenge is imposed by the difficulty of smart guessing as to the likely duration of the "harmful state" and the optimal path to recovery to the "preferred state." To be able to perform these additional functions effectively, the MLEP needs to have capability for "evaluative decision-making" which requires the higher level of cognitive recognition and assessment typically observed with human brain activity subjected to a situate metacognitive learning environment (see for example Tuzun, 2020a).

\section{DISCUSSION OF POTENTIAL HYBRID ENERGY PROCESS PLANT APPLICATIONS WITH MLEP}

It is appropriate to consider the potential use of the MLEP with industrial plants engaged in (i) synthetic chemicals/materials production, (ii) bio-chemicals/biomaterials extraction from natural and agricultural products, and (iii) gas and liquid fuels production. Clearly, a hybrid energy process plant in the first two categories will also have options to exercise with regards to combined cycle electrical power generation using heat recovery cycles whereas the plants engaged primarily in fuel production will increasingly want to exercise options to produce hybrid fuel mixes by dilution of high carbon content of fossil fuels with bioextracted organics, synthetic organic byproducts and hydrogen (see for example Holm-Nielsen and Ehimen, 2014; Staffell et al., 2019). Furthermore, processes involving anaerobic digestion of agricultural waste (see Dooms et al., 2018) are also in compliance with the requirements to eliminate waste through regeneration and re-use; refer to Figure $\mathbf{1}$ for green process applications. Hence, with both strategies of hybrid energy process applications, the aim is to provide reduced greenhouse gas emissions such as $\mathrm{CO}_{2}$ and $\mathrm{NO}_{x}$ whilst also reducing atmospheric pollutants such as VOCs and particulate emissions.

It is possible that the HEPP plants will able to use their own process waste as fuel if not entirely on their own but with integration into hybrid processes that will generate energy as well as energy recovery and material products from waste (see for example Al-Atta et al., 2018).

MLEP applications (refer to section "Previous Developments with CNN Applications"), within this environmental compliance background, can be utilized to demonstrate two primary routes for environmental impact Correction and Prevention (CAPA) (see Corrective Preventive Actions [CAPA] Guidelines, 2012): (1) "In-plant" Environmental Compliance Monitoring and Control of Emission Levels; and (2) "In-process" Abatement of Harmful Emissions. It can be argued that both strategies can be used simultaneously whilst the abatement schemes can be also factored into new plan designs and retrofits based on the experience gained with dynamic compliance monitoring and control of harmful emissions.

\section{Potential Role of MLEP With Integrated Carbon Capture and Storage (CCS) Schemes}

Capture and storage of $\mathrm{CO}_{2}$ in industrial plants is advocated to be a much needed principal technology development to combat climate change by reducing drastically the atmospheric emissions of $\mathrm{CO}_{2}$ that are shown scientifically to be responsible for global warming and its associated adverse environmental impacts (see for example Mc Sweeney et al., 2009; IPCC, 2014). This mandate has led to the development of power production integrated with $\mathrm{CO}_{2}$ capture. Recent examples of new technology developments include chemical looping combustion (CLC) with dual circulating fluidized beds (see Arnaiz del Pozo et al., 2019) and internally circulating fluid bed reactor (ICR) which 


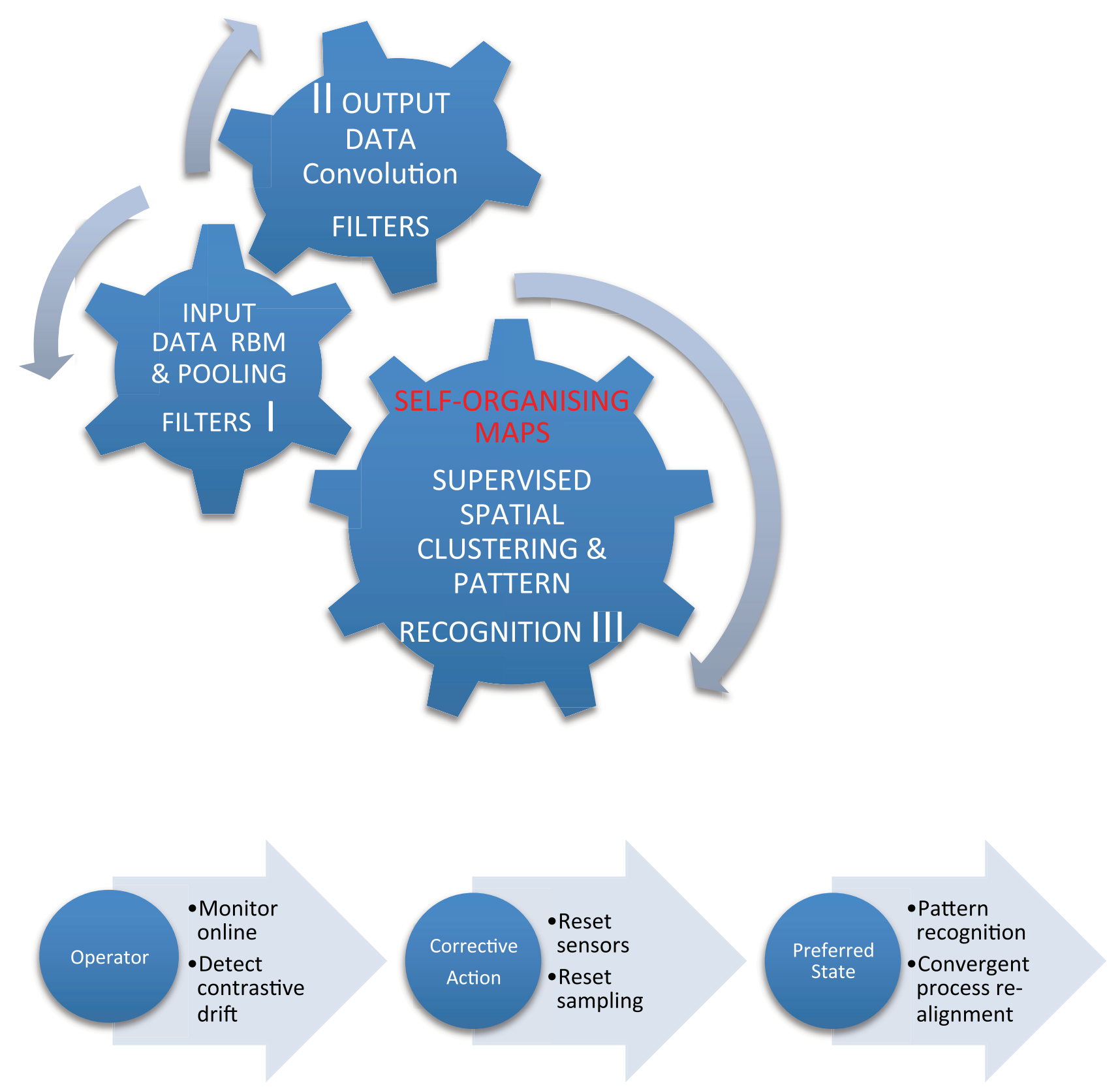

FIGURE 4 | KALEIDOSCOPE function of MLEP with dynamic operator interface.

combine natural gas reduction by air with particulate oxygen carrier (typically $\mathrm{NiO}$ particles supported on $\mathrm{Al}_{2} \mathrm{O}_{3}$ ) circulation to achieve conversion to $\mathrm{CO}_{2}$ (see Cloete et al., 2019). Common to these recent technology developments, is the desire to minimize the additional energy penalty of $\mathrm{CO}_{2}$ capture and storage, by integrating it fully within the combined power generation of the natural gas combined cycle (NGCC) plants.

IEA Sustainable Development Scenario (see International Energy Agency [IEA], 2018) assumes $\mathrm{CO}_{2}$ prices of $\$ 63$ per ton in 2025 and $\$ 140$ per ton in 2040. It is expected that the above hybrid energy technology developments will bring the price down to $\$ 50$ and below by avoiding separate energy intensive carbon capture process "add-on" s to existing power plants. However, the successful implementation of these most recent technologies depends on the ability to avoid $\mathrm{CO}_{2}$ leaks from the fuel section to the air section; reducing the $\mathrm{CO}_{2}$ capture efficiency of the reactor unit, while also avoiding $\mathrm{N}_{2}$ leaks from the air section to the fuel section; reducing the purity of the $\mathrm{CO}_{2}$ captured.

Furthermore, recent studies also show that scale up of a single ICR reactor unit even by $20 \%$ by size can exasperate the above leaks sufficiently to reduce the purity of captured $\mathrm{CO}_{2}$ from 96 to $93 \%$ (see Cloete et al., 2019). There is also the increased cost 
of handling elutriated solids with the increased reactor size (see Argyle and Bartholomew, 2015). The most important criteria for designing and operating the ICR units is therefore to minimize the amount of gas leakage between the reactor sections whilst maintaining an optimal rate of oxygen carrying solids circulation to ensure that the fuel is completely converted.

With both the CLC and ICR technology developments, it is imperative that the process efficiency and product purity is optimized through the use of a multiplex digital platform that can produce dynamic corrective action by periodic readjustment of the process parameters that will minimize contaminating gas leaks during gas switch and excessive solids elutriation. Here, it may be possible to develop supervised deep-learning strategies that will facilitate pattern recognition in time-series of multiple sensor data sets. This will in turn, allow for the operator fine-tuning, to achieve the "preferred state" that will maximize both the captured $\mathrm{CO}_{2}$ purity and the percentage of $\mathrm{CO}_{2}$ captured. It is believed that the wider implementation of these technologies depends greatly in their ability to utilize multivariate dynamic process control with fast convolutional neural network techniques described above so that significant fluctuations of captured $\mathrm{CO}_{2}$ purity and capture efficiency could be avoided. Due to the highly cyclic nature of the gas switch and solids circulation operations, the conventional steady-state set point process control techniques will prove inadequate to achieve the marginal process gains required with increased plant capacity.

\section{CONCLUSION}

The current studies relating to the use of field sensors and temporal and spatial data gathering for environmental compliance purposes in chemical and material processing plants are found to yield large and complex data sets. These can be analyzed effectively using "deep-learning" methods and systems of statistical analysis similar to those already developed for applications as diverse as tomographic imaging, medical sensor signal trace analysis and automatic speech recognition to name but a few.

The significance threshold of cross-correlations are established and ranked by first pre-pruning of data for bias and random error using statistical regression. Harvested data sets are subsequently analyzed using a digital processor platform that comprises as a multi-layer auto-encoder and a convolutional neural network decoder. Once operator defined "preferred state" is established for the process inputs and outputs, then process monitoring is carried out within a digital framework that calculates significance of divergence and convergence to and from the preferred-state in pre-selected temporal sequences for different spatial neighborhoods (emission bubbles) as a measure of the environmental compliance of the process operations.

A MLEP model considered can facilitate automated machine-learning; using a novel kaleidoscopic procedure for synchronizing input and output data convolution cycles, pattern recognition algorithms and a dynamic operator interface. Current $\mathrm{R} \& \mathrm{D}$ program in progress has so far constructed model MLEPs for two and three hidden-layer connectivity applications of solids handling and processing applications in a cereal processing plant (see Tuzun, 2018) and a research paper is underway to present the initial results. A similar project is being kick-started with a pharmaceutical process application based on the previous work of Fujiwara et al. (2005). Alongside the development of neural network algorithms for process plant applications, an interactive matrix of $[4,2]$ operating task algorithms are constructed to facilitate data training in 4 different variability modes within two different "in-plant" operation domains; refer to section "Real-Life Training and Plant Trials with MLEP” and Figure 3B.

The potential use of a digital MLEP multiplex platform is demonstrated to be ideally suited for the operations of hybrid energy process plants (HEPP) for chemicals and materials production; specifically for "in-plant" monitoring of environmental emissions and "in-process" control and abatement. Similar opportunities are illustrated in the operation of $\mathrm{CO}_{2}$ capture and storage schemes wholly integrated into process operations of combined cycle power generation plants. Further work will focus on the application of trained algorithms for environmental process monitoring and control using "reallife" plant data sets.

\section{DATA AVAILABILITY STATEMENT}

All datasets generated for this study are included in the article/supplementary material, further inquiries can be directed to the corresponding author.

\section{AUTHOR CONTRIBUTIONS}

The author is the sole contributor to the manuscript unless otherwise stated in the text.

\section{FUNDING}

Partial travel funding (2017-2018) was provided by the IChemE, United Kingdom; and EFCE Working Party for Mechanics of Particulate Solids for attendance at EFCE meetings and international conferences. Further conference attendance funding was received from University of Edinburgh (2017) and Oxford University (2016), United Kingdom. The R\&D activity is also supported by a non-commercial feasibility study (2019-) in California, United States.

\section{ACKNOWLEDGMENTS}

We would like to acknowledge preliminary discussions (20152016) with staff of Xenesis Ltd. at Surrey Technology Centre, Surrey Research Park, Guildford, Surrey, United Kingdom. Further discussions with and input from colleagues at IChemE UK Energy Centre (2018-2019) and EFCE Energy Section are also gratefully acknowledged. 


\section{REFERENCES}

Al-Atta, A., Huddle, T., Rodriguez, Y. G., Mato, F., Garcia-Serna, J., Cocero, M. J., et al. (2018). A techno-economic assessment of the potential for combining supercritical water oxidation with "in-situ" hydrothermal synthesis of nanocatalysts using a counter-current mixing reactor. Chem. Eng. J. 344, 431-440. doi: 10.1016/j.cej.2018.03.058

Antoniades, A., Spyrou, L., Martin-Lopez, D., Valentin, A., Alarcon, G., Sanei, S., et al. (2018). Deep neural architectures for mapping scalp to intracranial EEG. Int. J. Neural Syst. 28:1850009. doi: 10.1142/s0129065718500090

Antoniades, A., and Took, C. C. (2016). "Speeding up feature selection: a deepinspired network pruning algorithm, (2016)," in International Joint Conference on Neural Networks (IJCNN), 24-29 July 2016, Vancouver, British Columbia, doi: 10.1109/IJCNN.2016.7727221

Argyle, M. D., and Bartholomew, C. H. (2015). Heterogeneous catalyst activation and regeneration: a review. Catalysts 5, 145-269. doi: 10.3390/catal5010145

Arnaiz, del Pozo, C., Cloete, S., Cloete, J. H., Jimenez-Alvaro, A., and Amini, S. (2019). The potential of chemical looping combustion using the gas switching concept to eliminate the energy penalty of $\mathrm{CO} 2$ capture. Int. Greenhouse Gas Control 83, 265-281. doi: 10.1016/j.ijggc.2019.01.018

Bent, E. (2015). The Ground Exhales: Reducing Agriculture's Greenhouse Gas. Guelph, ON: University of Guelph. Available online at: www.theconversation.com

Cloete, J. H., Khan, M. N., Cloete, S., and Amini, S. (2019). Simulation-based design and economic evaluation of a novel internally circulating fluidised for power production with CO2 capture. Processes 7:723. doi: 10.3390/pr7100723

Corrective and Preventive Actions (Capa) Guidelines, (2012). Corrective and Preventive Actions (CAPA) Guidelines. Available at: www.rmbimedical.com/RegulatoryAffairs/capaguidelines.pdf.

Dooms, M., Benbelkacem, H., and Buffiere, P. (2018). High solid temperature phased anaerobic digestion from agricultural wastes: putting several reactors in sequence. Biochem. Eng. J. 130, 21-28. doi: 10.1016/j.bej.2017.11.011

Erickson, L. E., and Brase, G. (2019). Reducing Greenhouse Gas Emissions and Improving Air Quality - Two Interrelated Global Challenges. CRC Press: Taylor \& Francis.

European Environment Agency [EEA] (2016). "National emissions ceilings directive," in Clean Air Programme, Directive (EU) 2016/2284 [Copenhagen: European Environment Agency (EEA)].

Fujiwara, M., Zoltan, N. K., Chew, J. W., and Braatz, R. D. (2005). First principles and direct design approaches for the control of pharmaceutical crystallization. J. Process Control 15, 493-504. doi: 10.1016/j.jprocont.2004.08.003

Hafner, S. D., Howard, C., Muck, R. E., Roberta, B. F., Montes, F., Green, G. G., et al. (2013). Emission of volatile organic compounds from silage: compounds, sources, implications. Atmospheric Environ. 77, 827-839. doi: 10.1016/j.atmosenv.2013.04.076

Haykin, S. O. (2008). Neural Networks and Learning Machines: A Comprehensive Foundation, 3rd Edn. Mississauga, ON: Pearson International.

Holm-Nielsen, J. B., and Ehimen, E. A. (2014). "4-biorefinery plant design, engineering and process optimization," in Advances in Biorefineries: Biomass and Waste Supply Chain Exploitation, ed. K. Waldron, (Sawston: Woodhead Publ), 89-111. doi: 10.1533/9780857097385.1.89

IEAGHG, (2019). CCS in Climate Change Scenarios. Cheltenham: IEA Greenhouse Gas R\&D Programme.

International Energy Agency [IEA], (2018). World Energy Outlook. Paris: IEA.

IPCC, (2014). Fifth Assessment Report: "Mitigation of Climate Change". Cambridge: Intergovernmental Panel on Climate Change.

Jia, Z., and Julian, D. J. (2014). Synchronization of Signals for Multiple Data Sinks. United States Patent 9877130

Kulp, K., and Ponte, J. G. Jr. (eds) (2000). Handbook of Cereal Science and Technology, 2nd Edt. Edn. Boca Raton, FL: CRC Press.

Langkvist, M., Karlsson, L., and Loutfi, A. (2014). A review of unsupervised feature learning and deep learning for time-series Modeling. Pattern Recognit. Lett. 42, 11-24. doi: 10.1016/j.patrec.2014.01.008

Lou, H. L. (1995). Implementing the viterbi algorithm. IEEE Signal Process. 12, 42-52. doi: 10.1109/79.410439

Markiewicz, M. T. (2006). "Mathematical modeling of the heavy gas dispersion," in Models and Techniques for Health and Environmental Hazard Assessment Part 2: Air Quality Modeling, ed. M. Borysiewicz, (Otwock-Swierk: Institute of Atomic Energy), 279-302.
Mc Sweeney, C., New, M., and Lizcano, G. (2009). Climate Change Country ProfilesUK. Oxford: Oxford University, School of Geography and Environment, Tyndall Centre for Climate Change Research, Report Commissioned by British Council, RMetS and RGS-IBG.

Mendoza, J. M. F., Sharmina, M., Gallego-Schmid, A., Heyes, G., and Azapagic, A. (2017). Integrating backcasting and eco-design for the circular economy: the BECE framework. J. Indus. Ecol. 21, 526-544. doi: 10.1111/jiec. 12590

Rymarczyk, T., Klosowski, G., and Kozlowski, E. (2018). A non-destructive system based on electrical tomography and machine-learning to analyze moisture of buildings. Sensors 18:2285. doi: 10.3390/s18072285

Smith, R. (2005). Chemical Process: Design and Integration. Hoboken, NJ: John Wiley \& Sons Ltd.

Snyder, D., Garcia-Romero, D., and Povey, D. (2015). "Time delay deep neural network-based universal background models for speaker recognition," in IEEE Transactions, IEEE Workshop on Automatic Speech Recognition and Understanding (ASRU) (New York, NY: IEEE).

Staffell, I., Scamman, D., Velazquez-Abad, A., Balcombe, P., Dodds, P. E., Ekins, P., et al. (2019). The role of hydrogen and fuel cells in the global energy system. Energy Environ. Sci. 12, 463-491.

Sun, Q., Choi, W.-J., Jeffrey, M. G., and Tehrani, A. M. (2015). MIMO System and Method, US-Patent: US8989294 B2 (March 2015). San Diego, CA: Qualcomm Inc.

Tisserant, A., Pauliuk, S., Merciai, S., Schmidt, J., Fry, J., Wood, R., et al. (2017). Solid waste and the circular economy: a global analysis of waste treatment and waste footprints. J. Indus. Ecol. 21, 628-640. doi: 10.1111/jiec.12562

Tuzun, U. (2017). "Quality monitoring and control in breakfast cereal processes using neural network convolution of dynamic process data," in International Conference on Processing, Handling and Characterization of Particulate Materials (PARMAT 2017), Barcelona, 2476.

Tuzun, U. (2018). "Quality monitoring and control of air-borne particulates and VOCs emissions using neural network convolution of dynamic process data," in Proceedings of the 6th International Conference on Green Process Engineering, 3-6 June 2018, Toulouse, 76.

Tuzun, U. (2020a). Introduction to systems engineering and sustainability part i: student-centred learning for chemical and biological engineers. Educ. Chem. Eng. 31, 85-93. doi: 10.1016/j.ece.2020.04.004

Tuzun, U. (2020b). Introduction to systems engineering and sustainability part ii: interactive teaching of sustainability modeling by systems integration design for chemical and biological engineers. Educ. Chem. Eng. 31, 94-104. doi: 10.1016/j.ece.2020.04.005

Wenda-Piesik, A., Piesik, Ligor, T., and Buszewski, B. (2010). VOCs from cereal plants infested with crown rot: their identity and their capacity for inducing production of VOCs in uninfested plants, PEST. Int. J. Pest Manage. 56, 377-383. doi: 10.1080/09670874.2010.505668

Wu, Y. (2006). "A trellis connectivity analysis of random linear network coding with buffering," in Proceedings of the 2006 IEEE International Symposium on Information Theory, Seattle, WA, 768-772.

Yue, Q., Xu, X., Hillier, K., Cheng, K., and Pan, G. (2017). Mitigating greenhouse gas emissions in agriculture: from farm production to food consumption. J. Clean. Product. 149, 1011-1019. doi: 10.1016/j.jclepro.2017.02.172

Zare, V., and Mahmoudi, S. M. S. (2015). A thermodynamic comparison between organic rankine and kalina cycles for waste heat recovery from the gas turbine modular helium reactor. Energy 79, 398-406. doi: 10.1016/j.energy.2014. 11.026

Zhang, H., Zao, H., Li, Z., and Hu, E. (2019). Optimization potentials for the waste heat recovery of a gas-steam combined cycle power plant based on absorption heat pump. J. Therm. Sci. 28, 283-293. doi: 10.1007/s11630-0181055-7

Conflict of Interest: The author declares that the research was conducted in the absence of any commercial or financial relationships that could be construed as a potential conflict of interest.

Copyright (C) 2020 Tuzun. This is an open-access article distributed under the terms of the Creative Commons Attribution License (CC BY). The use, distribution or reproduction in other forums is permitted, provided the original author(s) and the copyright owner(s) are credited and that the original publication in this journal is cited, in accordance with accepted academic practice. No use, distribution or reproduction is permitted which does not comply with these terms. 